\title{
The Detection of Sentinel Nodes in Ovarian Cancer: A Feasibility Study
}

\author{
Marjolein Kleppe ${ }^{1}$, Boudewijn Brans ${ }^{2}$, Toon Van Gorp ${ }^{1,3}$, Brigitte F.M. Slangen ${ }^{1,3}$, Arnold J. Kruse ${ }^{1,3}$, Ivo N.A Pooters ${ }^{2}$, \\ Maartje G. Lotz ${ }^{2}$, Koen K. Van de Vijver ${ }^{4}$, and Roy F.P.M. Kruitwagen ${ }^{1,3}$ \\ ${ }^{I}$ Department of Obstetrics and Gynaecology, Maastricht University Medical Centre, Maastricht, The Netherlands; ${ }^{2}$ Department \\ of Nuclear Medicine, Maastricht University Medical Centre, Maastricht, The Netherlands; ${ }^{3}$ GROW, School for Oncology and \\ Developmental Biology, Maastricht, The Netherlands; and ${ }^{4}$ Department of Pathology, Maastricht University Medical Centre, \\ Maastricht, The Netherlands
}

\begin{abstract}
Few sentinel node (SN) studies in ovarian cancer have been reported, mainly because of the risk of tumor dissemination associated with the injection of tracers into the ovarian cortex. To our knowledge, the injection of tracers into the ovarian ligaments has not been explored. The aim of this study was to determine the feasibility of the SN procedure in ovarian cancer with tracer injection into the ovarian ligaments and to establish whether the procedure is safe for the healthcare workers. Methods: The study included patients who were at high risk of ovarian malignancy. Blue dye and radioactive colloid were injected into the proper ovarian ligament and suspensory ligament of the ovary. To measure professional radiation exposure, ring dose meters were worn by the surgeon, theater nurse, and pathologist during 3 procedures. Results: An SN procedure was performed in 21 patients, and at least $1 \mathrm{SN}$ location was identified in all patients using the y probe before retroperitoneal exploration. SNs were located in the paraaortic and paracaval regions only in $67 \%$ of the patients, in the pelvic region only in $9 \%$, and in both the paraaortic/paracaval and the pelvic regions in $24 \%$. All but 2 SNs were found on the ipsilateral side. In 6 patients who underwent retroperitoneal exploration, 1-4 SNs were identified using the $y$ probe and resected. Blue-stained SNs were detected in 2 patients. Positive SNs were detected in 1 patient with lymph node metastases. The amount of radiation exposure to the surgeon, theater nurse, and pathologist did not exceed the safe limit. Conclusion: The identification of SNs in all cases suggests that the $\mathrm{SN}$ procedure performed by injection of tracers in the ovarian ligaments is feasible and promising. The procedure is safe for the involved personnel. Further investigation is necessary to determine the clinical application of this new technique.
\end{abstract}

Key Words: sentinel node; ovarian cancer

J Nucl Med 2014; 55:1799-1804

DOI: 10.2967/jnumed.114.144329

$\mathbf{E}$

pithelial ovarian cancer (EOC) is the leading cause of death among gynecologic malignancies, and the high mortality rate is partially attributed to the fact that up to $75 \%$ of patients are di-

Received Jun. 16, 2014; revision accepted Sep. 4, 2014.

For correspondence or reprints contact: Roy F.P.M. Kruitwagen, Maastricht University Medical Centre, P.O. Box 5800, 6202 AZ, Maastricht, The Netherlands.

E-mail: r.kruitwagen@mumc.nl

Published online Oct. 20, 2014.

COPYRIGHT (C 2014 by the Society of Nuclear Medicine and Molecular Imaging, Inc. agnosed with advanced-stage EOC. In patients with clinical earlystage EOC, a staging procedure is recommended, which includes a total abdominal hysterectomy with bilateral salpingo-oophorectomy, omentectomy, numerous peritoneal biopsies, and a pelvic and paraaortic lymph node dissection. Although the International Federation of Obstetrics and Gynaecology (FIGO) recommends a complete pelvic and paraaortic lymphadenectomy, the extent of lymph node dissection differs greatly from center to center (1-3).

In patients with FIGO stage I-IIa ovarian cancer, adjuvant chemotherapy could be omitted after a comprehensive staging procedure. Adjuvant chemotherapy is obligatory in patients with FIGO stage IIbIV ovarian cancer. The omission of an adequate lymphadenectomy in clinical early-stage disease results in the underdiagnosis of stage III disease in approximately $10 \%-20 \%$ of patients, leading to the exclusion of adjuvant chemotherapy with curative intent $(4,5)$.

Lymphatic metastases of EOC mainly occur in the paraaortic and paracaval lymph nodes, although they can also be found in the pelvic lymph nodes (5-10). Tumor cells travel along the lymph vessels that accompany the ovarian artery and vein in the suspensory ligament up to the high paraaortic and paracaval regions. In pelvic lymph node metastasis, tumor cells are likely to move along lymph vessels in the proper ovarian ligament to the parauterine vessels in the broad ligament and further toward the iliac vessels. Therefore, lymph nodes need to be removed from different anatomic regions (the paraaortic and paracaval regions; common, internal, and external iliac vessels; and obturator fossa). The detection of metastases is positively correlated with the number of lymph nodes removed $(11,12)$. Complete pelvic and paraaortic lymphadenectomy was shown to result in the detection up to 250 or more lymph nodes (9). An overall incidence of lymph node metastasis of approximately $14 \%$ was reported in clinical early-stage EOC, with a higher incidence in grade III tumors $(20 \%)$ and in the serous histologic subtype (23\%) than in grade I (4\%) and mucinous tumors (3\%) (5). Because positive lymph nodes can be missed during lymph node sampling, systematic lymphadenectomy is considered the gold standard. However, this is a radical procedure that is associated with obvious morbidity, including nerve and vessel injury, increased blood loss, increased operating time, and the formation of lymphocysts and lymphedema $(10,12,13)$.

The sentinel node $(\mathrm{SN})$ procedure enables the identification of the first lymph node receiving primary lymphatic flow (the socalled SN). Histopathology results indicate the nodal status of all lymph nodes in a certain anatomic region. The detection of a negative SN suggests that the remaining lymph nodes are not involved. As a consequence, the patient may be spared a radical 
lymphadenectomy and thus the associated morbidity. The SN technique has been proven effective in vulvar and breast cancer, and SN studies in cervical and uterine cancers are currently under way (14-17). For the ovary, SN studies are scarce, partly because of the risk of tumor dissemination associated with the injection of tracers into the ovarian cortex (16-19).

In the current study, we examined the effect of tracer injection into the ovarian ligaments instead of the ovarian cortex during the $\mathrm{SN}$ procedure.

The objective of this technique was to prevent spillage of cyst fluid and malignant cells and to inject the tracer near the draining lymph vessels in the ovarian ligaments. Moreover, this technique enables the repeated injection of the tracer in the same location, irrespective of the size of the ovarian mass. To the best of our knowledge, the injection of tracers into the ovarian ligaments has not been explored previously. The aim of the present study was to determine the feasibility of the SN procedure performed by the injection of tracers into the ovarian ligaments and to establish whether the procedure is safe for the healthcare workers.

\section{MATERIALS AND METHODS}

\section{Patients}

Patients diagnosed with a pelvic mass suggestive of a malignant ovarian tumor who were treated in Maastricht University Medical Centre were eligible for inclusion in the study (20). All patients provided fully informed consent before enrollment in the study, and the protocol was approved by the Local Ethics Committee (approval number NL40323.068.12) (clinical trial registration number NCT01734746). Exclusion criteria were as follows: previous surgery on one or both ovaries; previous vascular surgery of the aorta, caval vein, or iliac vessels; previous lymphadenectomy or lymph node sampling in the iliac or paraaortic region; a history of malignant lymphoma; a history of a malignant tumor in the abdominal cavity; previous allergic reaction to blue dye or human albumin; and pregnant or lactating patients.

\section{SN Procedure}

After opening of the abdomen and before removal of the enlarged and suggestive ovary, blue dye and the radioactive isotope were injected on the dorsal and ventral side of the proper ovarian ligament and the suspensory ligament, close to the ovary and just underneath the peritoneum (Fig. 1). Each of the 4 injections contained $0.2-0.5 \mathrm{~mL}$ of blue dye (Blue Patente V, $25 \mathrm{mg} / \mathrm{mL}$; Guerbet Nederland B.V.) and $20 \mathrm{MBq}$ of ${ }^{99 \mathrm{~m}} \mathrm{Tc}$-labeled albumin nanocolloid with a particle size less

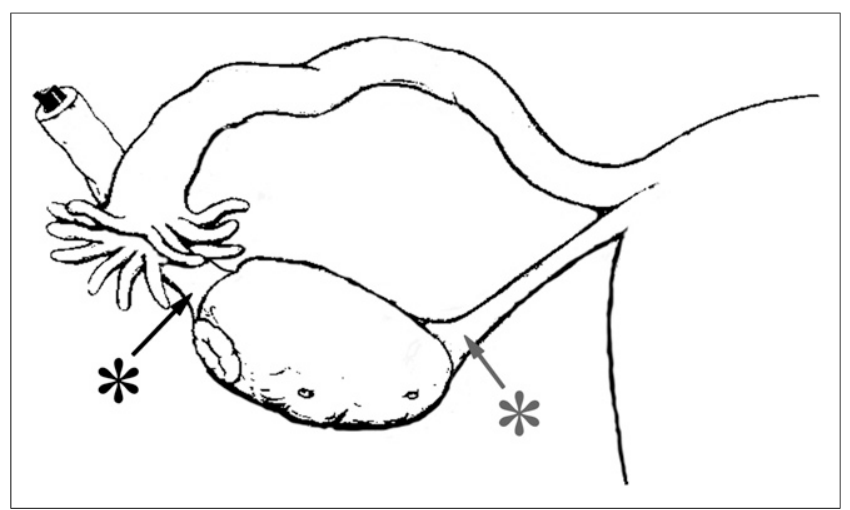

FIGURE 1. Location of injection of tracers. Tracers were injected on ventral and dorsal sides of both ligaments. Black asterisk = suspensory ligament of ovary; gray asterisk = proper ovarian ligament. than $80 \mathrm{~nm}$ ( ${ }^{99 \mathrm{~m}} \mathrm{Tc}$-nanocolloid or Nanocoll; GE Healthcare). After a 15-min interval, the adnexal mass was removed and sent to the pathologist for the examination of frozen sections, followed by examination of the different lymph node regions for the presence of SNs using a standard 12-mm straight $\gamma$ probe (Node Seeker; Intra Medical Imaging). The location was considered positive if the counts were at least 10 -fold higher than the background radiation.

If a benign or borderline ovarian tumor was detected, lymph node dissection was not performed. Nevertheless, in these patients potential SNs were once more localized transperitoneally using the $\gamma$ probe. Six different regions were used to describe the localization of the hot spots with the $\gamma$ probe as follows: upper and lower paraaortic regions separated by the origin of the inferior mesenteric artery, upper and lower paracaval regions, and right and left pelvic regions.

On identification of a malignant mass, the retroperitoneal space was opened, and the SNs were localized with the $\gamma$ probe or visually (blue dye). The surgeon recorded the number and location of the resected SNs, with 12 different regions used to describe their localization as follows: upper and lower paraaortic regions, upper and lower interaortocaval regions, upper and lower paracaval regions, right and left common iliac regions, right and left external iliac regions, and right and left obturator fossa regions. After removal of the SNs, the location from which they were removed was reexamined for the presence of radioactivity. If the radioactivity was less than $10 \%$ of that in the initial positive lymph node, no further action was taken. After removal of the SNs, a complete standard staging procedure was performed including a comprehensive sampling of other lymph nodes in the different anatomic locations. The surgeon also recorded the location of the removed non-SNs.

\section{Histopathology}

SNs and non-SNs were examined separately. Non-SNs were cut into single sections and stained with hematoxylin and eosin (H\&E), according to the standard protocol for lymph node examination. SNs were cut into 2-mm sections for H\&E staining. H\&E-negative SNs in the first section were further cut into $500-\mu \mathrm{m}$ sections and analyzed for the presence of micrometastases $(<2 \mathrm{~mm})$. At each step, immunohistochemical staining with cytokeratin MNF-116 was performed. Metastases greater than $2 \mathrm{~mm}$, those between 0.2 and $2 \mathrm{~mm}$, and those less than $0.2 \mathrm{~mm}$ were considered macrometastases, micrometastases, and isolated tumor cells, respectively.

\section{Sample Size Calculation}

A sample size of 20 evaluable patients was considered large enough to determine whether identification of SNs is feasible by injection of tracers into the ovarian ligaments.

\section{Measurement of Radiation Exposure to Personnel}

To measure professional radiation exposure, the radiation dose to which the surgeon, theater nurse, and pathologist were exposed during 3 procedures was measured. A ring dose meter, pointed toward the inside of the hand, was worn by these persons at the base of the index finger of the right and left hand during surgery or handling of tissues. Additionally, electronic dose meters were placed on the body at chest level.

\section{RESULTS}

\section{Patients' Characteristics}

Twenty-two patients were included in the study. The SN procedure was successfully completed in 21 of 22 patients (96\%). In 1 patient with a dermoid cyst, the high number of adhesions prevented access to the ovarian ligaments without interfering with the normal anatomy during injection of the tracer. Allergic or adverse reactions did not occur in any of the patients. The SN procedure was performed 7 times 
on the left side and 14 times on the right side. The final pathology results showed that 7 patients had a benign tumor, 8 had a borderline tumor, and 6 had malignant EOC. All frozen sections were correctly diagnosed and confirmed in the final pathology results. One patient was diagnosed with a primary ovarian tumor and endometrial cancer (grade I endometrioid type tumor with minimal myometrial invasion). On the basis of the staging procedure, intraabdominal tumor metastases were not identified in any of these patients.

\section{Transperitoneal SN Detection with y Probe After Injection of Tracer}

In all patients, at least 1 hot spot could be identified with the $\gamma$ probe within $15 \mathrm{~min}$ after injection of the radioactive tracer. Hot spots were found only in the paraaortic and paracaval regions in $67 \%$ of the patients, in the pelvic region only in $9 \%$, and in both the paraaortic/paracaval and the pelvic regions in $24 \%$ (Table 1). Eight patients had more than 1 hot spot ( 2 hot spots in 7 and 4 hot spots in 1 patient). All but 2 of the $31 \mathrm{SN}$ locations were on the ipsilateral side (Fig. 2A). Nearly all paraaortic SNs related to the left ovary were situated just below the renal vein and above the level of the inferior mesenteric artery, whereas the paracaval SNs related to the right ovary were lower, being mainly located at the level of the inferior mesenteric artery.

\section{Retroperitoneal SN Detection (Table 2; Fig. 2B)}

Retroperitoneal exploration with lymph node dissection was performed in 6 patients. In 4 of these patients, additional SNs were detected with the $\gamma$ probe at the location of the hot spot during retroperitoneal exploration. Furthermore, after opening the peritoneum, additional SNs were identified in other regions in 3 patients. In all 6 patients, the SNs were clearly identified with the radioactive tracer using the $\gamma$ probe. In 2 patients (patients 1 and 5), the SNs also showed blue staining.

All paraaortic SNs related to the left ovary were situated just below the renal vein. The paracaval SNs related to the right ovary were mainly located at the level of the inferior mesenteric artery.

\section{Histopathology}

Eighteen tissue specimens were positive for SNs. In the final histologic examination, more than 1 lymph node was identified in 4 of these tissue specimens (Table 2; Fig. 2B).

In 1 patient (patient 5), metastases were found in the resected SNs. Three of 4 SNs removed contained micrometastases, which were also detected in 1 of the remaining 8 lymph nodes removed.

\section{Radiation Exposure to Personnel}

Of 3 procedures performed, only the dose meters at the extremities of the surgeon gave values above background radiation level. The total dose measured during 3 procedures was $0.06 \mathrm{mSv}$ at the dominant hand of the surgeon and $0.17 \mathrm{mSv}$ at the nondominant hand.

\section{DISCUSSION}

In the present study, we showed that perioperative injection of tracers into the ovarian ligaments is a feasible method for the detection of SNs. One or more SNs were detected with the $\gamma$ probe in all the patients $(n=21)$ who underwent the SN procedure. Hot spots were found isolated in the paraaortic and paracaval regions in most patients (67\%), in the pelvic region only in $9 \%$, and in both the paraaortic/paracaval and the pelvic regions in $24 \%$. In all but 2 patients, the SNs were ipsilateral to the injection site. These findings correlate well with the results of a review on the incidence and location of lymph node metastases in clinical stage I-II EOC, in which metastases were found isolated in the paraaortic region in $50 \%$ of the patients, in the pelvic region in $20 \%$, and in both the paraaortic and the pelvic region in the remaining $30 \%$ (5).

SN studies in ovarian cancer are scarce. Negishi et al. used injection of an activated charcoal solution into the ovarian capsule to identify SNs and reported the detection of SNs in the paraaortic region in all 11 patients analyzed (overall $18 \mathrm{SNs}$ ) and in the pelvic region in 4 patients (overall $4 \mathrm{SNs}$ ) (18). Nyberg et al. analyzed 16 patients with high-risk uterine cancer by injecting technetium and blue dye into the right or left ovary and detected $1-3$ SNs in 15 of the 16 patients (19). The SNs $(n=30)$ were all located in the paraaortic region. The SNs related to the left ovary were mainly $(64 \%)$ located above the level of the inferior mesenteric artery, whereas most SNs of the right ovary (94\%) were found below the level of the inferior mesenteric artery, which is in agreement with our findings. In both studies, the tracer was injected into the ovary. However, the injection of tracers into the ovary can be difficult when bulky ovarian masses are present. Furthermore, the injection of tracers into the ovarian capsule is associated with a potential risk of tumor dissemination $(16,17)$. This probably explains why, hitherto, SN detection in ovarian cancer has not been adopted in clinical practice. The adnexal mass should preferably be removed intact, without rupture. The spillage of malignant cells within the peritoneal cavity will increase the patient's stage. Although the effect on prognosis of preoperative spill still is controversial, current practice by the FIGO guidelines is to avoid spillage (21). In the present study, tracers were injected in the ovarian ligaments to overcome the risk of tumor dissemination. In only 1 of the 22 patients, we were unable to access the ovarian ligaments because of adhesions potentially caused by leakage of a dermoid cyst and not the size of the tumor.

TABLE 1

Transperitoneal SN Detection with y Probe

\begin{tabular}{|c|c|c|c|c|c|}
\hline \multirow[b]{2}{*}{ Tumor location } & \multirow[b]{2}{*}{ Histology } & \multirow[b]{2}{*}{ Number } & \multicolumn{3}{|c|}{ Hot spot location } \\
\hline & & & $\begin{array}{c}\text { Only } \\
\text { paraaortic/paracaval }\end{array}$ & Only pelvic & $\begin{array}{c}\text { Both paraaortic/paracaval } \\
\text { and pelvic }\end{array}$ \\
\hline \multirow[t]{3}{*}{ Ovary } & Benign & 7 & 6 & 0 & 2 \\
\hline & Borderline & 8 & 4 & 2 & 2 \\
\hline & Malignant & 5 & 3 & 0 & 1 \\
\hline Ovary and endometrium & Both malignant & 1 & 1 & 0 & 0 \\
\hline Total & & 21 & $14(67 \%)$ & $2(9 \%)$ & $5(24 \%)$ \\
\hline
\end{tabular}




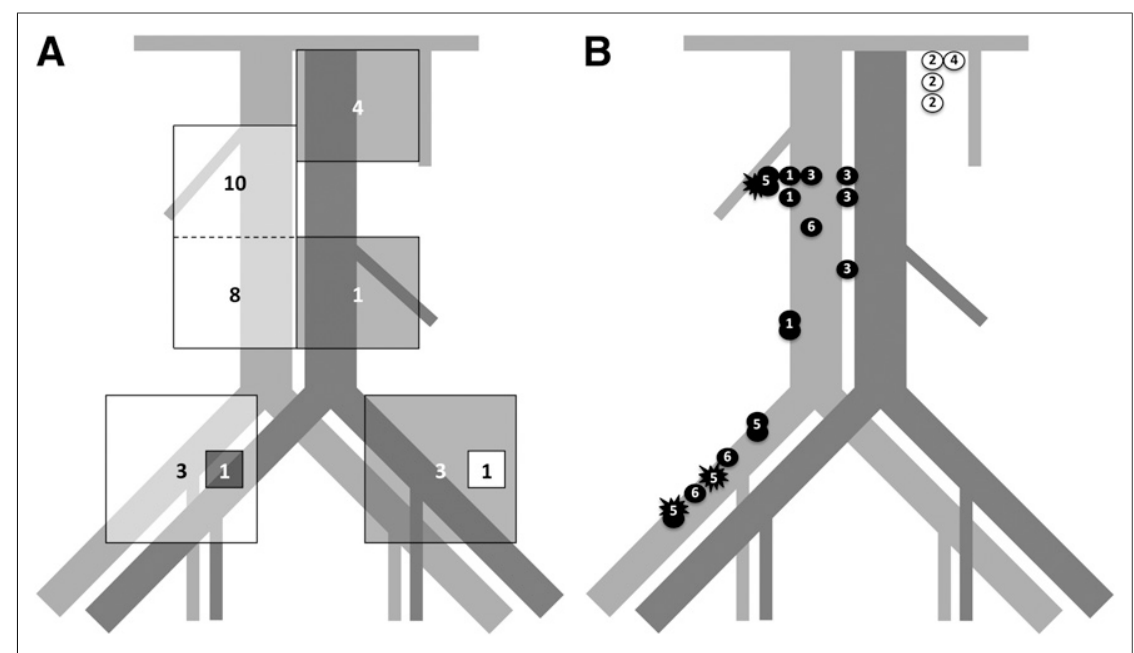

FIGURE 2. Detection of SNs. (A) Transperitoneal detection of hot spots. Figure shows location and number of hot spots found with y probe. White squares = location of hot spots found in patients $(n=14)$ with tumor on right side; gray squares = location of hot spots found in patients $(n=7)$ with tumor on left side. (B) Location of SNs at retroperitoneal exploration. Numbers correspond with patients' number as described in Table 2. Black circles = location of SNs found in patients with tumor on right side; black stars = location of SNs containing metastasis; white circles $=$ location of SNs found in patients with tumor on left side.

The exact timing for the detection of positive lymph nodes remains undefined. The time period should be long enough to allow the tracers to be transported to the SNs, but an increased lag time requires prolonged general anesthesia and increased costs. Therefore, delaying surgery for a period longer than 15 min was deemed not to be feasible in daily practice. Negishi et al. and Nyberg et al. waited for $10 \mathrm{~min}$ and 10-21 min after injection, respectively $(18,19)$. Intraoperative SN procedures in the case of cervical and endometrial cancer also use comparable time intervals (16). In the present study, we used a 15-min interval between injection of the tracers and resection of the adnexal mass, and at least $1 \mathrm{SN}$ was identified in all patients within this period. In the case of an ovarian malignancy, the time between injection of the tracers and detection and resection of the SNs was at least $50 \mathrm{~min}$ (including $15 \mathrm{~min}$ waiting time after injection, and time spent for removal of the ovarian tumor and frozen section analysis).
As described in the "Results" section, in 4 patients additional $\mathrm{SNs}$ within the same region were discovered after the opening of the retroperitoneum, and in 3 patients additional SNs were discovered in other regions. Identifying more lymph nodes during retroperitoneal exploration could be explained by a better and more precise accessibility of the lymph node locations but possibly also because of a longer time interval after injection of the tracer. Indeed, additional work needs to be done to optimize the $\mathrm{SN}$ detection technique in ovarian cancer. Items to be determined include the optimal colloid particle sizes, which results in the fastest migration and best retention in SNs. In a study by Glass et al., examining the kinetics of 3 different radioactive agents in cutaneous melanoma, detection at $30 \mathrm{~min}$ was more appropriate than late imaging (22). Paganelli et al., studying breast cancer, showed that the number of SNs detected strongly related to the size of the particle - that is, the smaller the particles, the greater the number of lymph nodes (23). However, all these data may be organ-dependent, so it is presently unknown if this is also the case in ovarian cancer. Moreover, newly developed hybrid SN detection agents, combining radioactivity with fluorescence or paramagnetic capabilities; alternative operative techniques such as multiphased injection; and new intraoperative imaging technologies may provide additional advantages (24-27).

In general, lymphatic mapping involves the injection of a blue dye or a radioactive isotope. SN studies in different primary tumors showed that the detection rate is highest when a radioactive isotope and the blue dye are used in combination $(28,29)$. In the present study, blue-stained SNs were identified in only 2 of 6 patients during retroperitoneal exploration. The long interval between the injection of the tracer and retroperitoneal exploration of at least $50 \mathrm{~min}$ may have caused the blue dye to fade before the surgical procedure. An SN study in cervical cancer reported the

TABLE 2

Overview of Patients with Lymph Nodes Removed

\begin{tabular}{|c|c|c|c|c|c|c|}
\hline \multirow[b]{3}{*}{ Patient no. } & \multirow[b]{3}{*}{ Transperitoneal detection, SN location } & \multicolumn{5}{|c|}{ Retroperitoneal detection } \\
\hline & & \multicolumn{3}{|c|}{ SNs } & \multicolumn{2}{|c|}{ Non-SNs } \\
\hline & & Location & No.* & Metastases & No. & Metastases \\
\hline 1 & 1 & 3 & 4 & 0 & 11 & 0 \\
\hline 2 & 1 & 3 & 3 & 0 & 7 & 0 \\
\hline 3 & 1 & 4 & 4 & 0 & 8 & 0 \\
\hline 4 & 1 & 1 & 1 & 0 & 20 & 0 \\
\hline $5^{\dagger}$ & 2 & 4 & 8 & 3 & 8 & 1 \\
\hline 6 & 2 & 3 & 3 & 0 & 11 & 0 \\
\hline
\end{tabular}


detection of blue-stained lymph nodes after a median time of 7 min after injection, with the lymph nodes remaining blue for a median time of $21 \mathrm{~min}$ (30). In future studies, the utility of the blue dye as a tracer in the case of ovarian cancer should be examined in more detail.

A pelvic and paraaortic lymphadenectomy or, at least, comprehensive lymph node sampling in both the pelvic and the paraaortic/ paracaval regions is an essential part of staging in clinical earlystage ovarian cancer. The presence of lymph node metastases is an indication for adjuvant chemotherapy. The main purpose of the $\mathrm{SN}$ technique in clinical early-stage ovarian cancer is the reduction of treatment-related morbidity in patients without metastatic lymph nodes while avoiding the risk of missing involved lymph nodes. Approximately $14 \%$ of patients with clinical early-stage disease have lymph node metastases (5). As a consequence, $86 \%$ of patients are not likely to benefit from a lymphadenectomy, although they are at risk for surgery-related morbidity. Complete pelvic or paraaortal lymphadenectomies are associated with increased peri- and postoperative morbidities, which mainly include lymphocysts and lymphorrhea $(10,12,13)$. Furthermore, the use of SN procedure as a substitute for a lymph node sampling may even be associated with a higher sensitivity for the identification of involved lymph nodes.

The SN procedure is already proven to be accurate and costeffective, related to a marked improvement in postoperative morbidity, in different types of cancer such as breast and vulvar (31-33). However, in patients with a complex adnexal mass, the SN procedure should be performed before the diagnosis EOC can be made on the basis of a frozen section. As a consequence, a certain number of patients obtain the $\mathrm{SN}$ procedure while having a benign adnexal mass. Therefore, the added costs of SN procedures in patients with a benign ovarian tumor should also be considered. For these patients, the SN procedure does not provide any advantages; however, the risk of complications and side effects of the procedure is low (34-36).

The radiation exposure measurements for the surgeon, theater nurse, and pathologist during 3 procedures showed values above background radiation level only for the surgeon. The maximum value, measured at the nondominant hand, was less than $0.1 \mathrm{mSv}$ per procedure. The difference between the dominant hand and the nondominant hand is in accordance with a previous study of de Kanter et al., who examined radiation exposure of personnel during an SN procedure in breast cancer (37). The difference between the dominant hand and the nondominant hand is caused by the operating technique. The nondominant hand presents the tissue to be removed by the dominant hand, resulting in a shorter distance between nondominant hand and radioactive tissue. The maximum hand dose for nonexposed and exposed workers is 50 and $500 \mathrm{mSv} / \mathrm{y}$, respectively, meaning that no dose limits are exceeded and there is no additional risk for the involved personnel, if the number of procedures remains below 500 procedures/person/y.

The present study has limitations. First, a limited number of patients were included in the analysis, mostly because ovarian cancers are often diagnosed at an advanced stage of the disease. And although SNs were detected in all 21 patients who received the injection of tracers, the high number of benign and borderline ovarian tumors resulted in the resection of lymph nodes in only a small group of patients. In planning the study design, we presumed that in approximately $60 \%$ of the patients with a clinical suspicion of ovarian tumor, a frozen section during surgery would be confirmatory. This calculation was based on the accuracy of the risk of malignancy index (sensitivity, 78\%; specificity, 87\%) and a prevalence of malignancies within our study population of $20 \%$ (38). However, 8 of 14 malignancies were defined as borderline tumors, in which case no lymph nodes are resected as part of the staging procedure. The numbers of cases in this study are too small to reach conclusions regarding false-negative SNs based on histology. Second, the 1 patient with lymph node metastasis had an ovarian and an endometrial tumor. The positive lymph nodes could be related to the synchronous endometrial tumor. Further investigation, including a larger number of patients in a multicenter study, is necessary to determine the clinical application of this innovative technique.

\section{CONCLUSION}

In the present study, we showed that the $\mathrm{SN}$ procedure performed through the injection of tracers into the ovarian ligaments is feasible and promising. The procedure is safe for the involved personnel. Injection of radioactive tracers resulted in the identification of SNs in all patients, suggesting that the technique could potentially be incorporated into routine clinical practice in patients with early-stage ovarian cancer. The SN procedure could be a cost-effective method to reduce the morbidity and rate of complications associated with complete pelvic and paraaortic lymphadenectomy. Plans for a multicenter prospective trial are currently under way to explore the benefits of the SN technique based on the injection of tracers in the ovarian ligaments in patients with clinical early-stage ovarian cancer.

\section{DISCLOSURE}

The costs of publication of this article were defrayed in part by the payment of page charges. Therefore, and solely to indicate this fact, this article is hereby marked "advertisement" in accordance with 18 USC section 1734 . No potential conflict of interest relevant to this article was reported.

\section{REFERENCES}

1. Benedet JL, Bender H, Jones H III, Ngan HY, Pecorelli S. FIGO staging classifications and clinical practice guidelines in the management of gynecologic cancers. FIGO Committee on Gynecologic Oncology. Int J Gynaecol Obstet. 2000;70:209-262.

2. Angioli R, Plotti F, Palaia I, et al. Update on lymphadenectomy in early and advanced ovarian cancer. Curr Opin Obstet Gynecol. 2008;20:34-39.

3. Di Re F, Baiocchi G. Value of lymph node assessment in ovarian cancer: status of the art at the end of the second millennium. Int J Gynecol Cancer. 2000;10: 435-442.

4. Piver MS, Barlow JJ, Shashikant BL. Incidence of subclinical metastases in stage I and II ovarian carcinoma. Obstet Gynecol. 1978;52:100-104.

5. Kleppe M, Wang T, Van Gorp T, Slangen BFM, Kruse AJ, Kruitwagen RFPM. Lymph node metastasis in stages I and II ovarian cancer: a review. Gynecol Oncol. 2011;123:610-614.

6. Onda T, Yoshikawa H, Yokota H, Yasugi T, Taketani Y. Assessment of metastases to aortic and pelvic lymph nodes in epithelial ovarian carcinoma: a proposal for essential sites for lymph node biopsy. Cancer. 1996;78:803-808.

7. Tsumura N, Sakuragi N, Hareyama H, et al. Distribution pattern and risk factors of pelvic and para-aortic lymph node metastasis in epithelial ovarian carcinoma. Int J Cancer. 1998;79:526-530.

8. Morice P, Joulie F, Camatte S, et al. Lymph node involvement in epithelial ovarian cancer: analysis of 276 pelvic and paraaortic lymphadenectomies and surgical implications. J Am Coll Surg. 2003;197:198-205.

9. Harter P, Gnauert K, Hils R, et al. Pattern and clinical predictors of lymph node metastases in epithelial ovarian cancer. In J Gynecol Cancer. 2007;17:12381244.

10. Ditto A, Martinelli F, Reato C, et al. Systematic para-aortic and pelvic lymphadenectomy in early stage epithelial ovarian cancer: a prospective study. Ann Surg Oncol. 2012;19:3849-3855.

11. Carnino F, Fuda G, Ciccone G, et al. Significance of lymph node sampling in epithelial carcinoma of the ovary. Gynecol Oncol. 1997;65:467-472. 
12. Maggioni A, Benedetti Panici P, Dell'Anna T, et al. Randomised study of systematic lymphadenectomy in patients with epithelial ovarian cancer macroscopically confined to the pelvis. Br J Cancer. 2006;95:699-704.

13. van de Poll-Franse LV, Pijnenborg JMA, Boll D, et al. Health related quality of life and symptoms after pelvic lymphadectomy or radiotherapy vs. no adjuvant regional treatment in early-stage endometrial carcinoma: a large populationbased study. Gynecol Oncol. 2012;127:153-160.

14. de Hullu JA, Hollema H, Piers DA, et al. Sentinel lymph node procedure is highly accurate in squamous cell carcinoma of the vulva. J Clin Oncol. 2000; 18:2811-2816.

15. Mansel RE, Fallowfield L, Kissin M, et al. Randomized multicenter trial of sentinel node biopsy versus standard axillary treatment in operable breast cancer: the ALMANAC Trial. J Natl Cancer Inst. 2006;98:599-609.

16. El-Ghobashy AE, Saidi SA. Sentinel node sampling in gynaecological cancers: techniques and clinical applications. Eur J Surg Oncol. 2009;35:675-685.

17. Giammarile F, Vidal-Sicart S, Valdés Olmos RA. Uncommon applications of sentinel lymph node mapping: urogenital cancers. Q J Nucl Med Mol Imaging. 2014;58:161-179.

18. Negishi H, Takeda M, Fujimoto T, et al. Lymphatic mapping and sentinel node identification as related to the primary sites of lymph node metastasis in early stage ovarian cancer. Gynecol Oncol. 2004;94:161-166.

19. Nyberg RH, Korkola P, Tech L, Mäenpää J. Ovarian sentinel node, is it feasible? Int J Gynecol Cancer. 2011;21:568-572.

20. Kleppe M, Van Gorp T, Slangen BFM, et al. Sentinel node in ovarian cancer: study protocol for a phase 1 study. Trials. 2013;14:47.

21. Prat J. Staging classification for cancer of the ovary, fallopian tube, and peritoneum. Int J Gynaecol Obstet. 2014;124:1-5.

22. Glass EC, Essner R, Morton DL. Kinetics of three lymphoscintigraphic agents in patients with cutaneous melanoma. J Nucl Med. 1998;39:1185-1190.

23. Paganelli G, De Cicco C, Cremonesi M, et al. Optimized sentinel node scintigraphy in breast cancer. $Q J$ Nucl Med. 1998;42:49-53.

24. Ahmed M, Purushotham AD, Douek M. Novel techniques for sentinel lymph node biopsy in breast cancer: a systematic review. Lancet Oncol. 2014;15:e351-e362.

25. Tsuchimochi M, Hayama K. Intraoperative gamma cameras for radioguided surgery: technical characteristics, performance parameters, and clinical applications. Phys Med. 2013;29:126-138.
26. Schaafsma BE, van der Vorst JR, Gaarenstroom KN, et al. Randomized comparison of near-infrared fluorescence lymphatic tracers for sentinel lymph node mapping of cervical cancer. Gynecol Oncol. 2012;127:126-130.

27. Schaafsma BE, Verbeek FP, Peters AA, et al. Near-infrared fluorescence sentinel lymph node biopsy in vulvar cancer: a randomised comparison of lymphatic tracers. BJOG. 2013;120:758-764.

28. Hayashida T, Jinno H, Sakata M, et al. Superiority of radioactive over blue dye for sentinel lymph node detection in breast cancer. Eur Surg Res. 2010;44: $111-116$.

29. Eiriksson LR, Covens A. Sentinel lymph node mapping in cervical cancer: the future? BJOG. 2012;119:129-133.

30. Levenback C, Coleman RL, Burke TW, et al. Lymphatic mapping and sentinel node identification in patients with cervix cancer undergoing radical hysterectomy and pelvic lymphadenectomy. J Clin Oncol. 2002;20:688-693.

31. Van der Zee AGJ, Oonk MH, De Hullu JA, et al. Sentinel node dissection is safe in the treatment of early-stage vulvar cancer. J Clin Oncol. 2008;26:884-889.

32. Verry H, Lord SJ, Martin A, et al. Effectiveness and cost-effectiveness of sentinel lymph node biopsy compared with axillary node dissection in patients with early-stage breast cancer: a decision model analysis. Br J Cancer. 2012;106: 1045-1052.

33. Schulze T, Mucke J, Markwardt J, Schlag PM, Bembenek A. Long-term morbidity of patients with early breast cancer after sentinel lymph node biopsy compared to axillary lymph node dissection. J Surg Oncol. 2006;93:109-119.

34. Bézu C, Coutant C, Salengro A, Daraï E, Rouzier R, Uzan S. Anaphylactic response to blue dye during sentinel node biopsy. Surg Oncol. 2011;20:e55-e59.

35. Dewachter P, Mouton-Faicre C, Trechot P, Lleu JC, Mertes PM. Severe anaphylactic shock with methylene instillation. Anesth Analg. 2005;101:149-150.

36. Rzymski P, Wozniak J, Opala T, Wilczak M, Sajdak S. Anaphylactic reaction to methylene blue dye after laparoscopic chromopertubation. Int J Gynaecol $\mathrm{Ob}$ stet. 2003;81:71-72.

37. de Kanter AY, Arends PPAM, Eggermont AMM, Wiggers T. Radiation protection for the sentinel node procedure in breast cancer. Eur J Surg Oncol. 2003; 29:396-399.

38. Geomini P, Kruitwagen RFPM, Bremer GL, Cnossen J, Mol BW. The accuracy of risk scores in predicting ovarian malignancy: a systematic review. Obstet Gynecol. 2009;113:384-394. 